\title{
European guidelines on resuscitation
}

\author{
Defibrillate as early and as often as possible
}

European policies are not usually simple, sensible, and practical. A welcome exception are the clear guidelines on managing cardiac arrest produced by the European Resuscitation Council through its working parties on basic and advanced life support. ${ }^{12}$ Abridged versions of these appear in this week's journal (p 1587, ${ }^{3}$ p $1589^{4}$ ).

Because defibrillation within 90 seconds of the onset of ventricular fibrillation is the treatment most likely to improve survival, the guidelines give priority to activating the emergency services and to the immediate delivery of a direct current shock. Survival from cardiac arrest in the community can be improved by adequately training the public and ensuring the prompt arrival of emergency services with facilities for defibrillation.

The guidelines for basic life support suggest that the initial management of an apparently lifeless casualty should include an assessment of the airway, breathing, and circulation before any active intervention takes place. If cardiac arrest is confirmed then the first action is to telephone for help. These substantial changes in the sequence of actions may pose some initial difficulty to teachers of basic life support who have well developed educational routines. The changes, however, are justified by the scientific and clinical literature and agree with similar guidelines recently issued in the United States. ${ }^{5}$ The guidelines clearly state that no cases of transmission of hepatitis B or HIV by mouth to mouth resuscitation have been reported.

The "precordial thump," which is given prominence in the advanced guidelines for life support, is not mentioned in the basic guidelines. This is surprising as it is most effective if delivered soon after the onset of an arrhythmia. ${ }^{6}$ The risk that the thump may convert pulseless ventricular tachycardia to ventricular fibrillation is surely outweighed by the possible benefits. Another omission from the basic guidelines is of a foreign body in the airway. This is a common and easily treated emergency that should be given prominence in teaching on basic life support.

The advanced guidelines retain the three main protocols found in previous guidelines-for ventricular fibrillation (including pulseless ventricular tachycardia), asystole, and electromechanical dissociation. ${ }^{7}$ The organisation of the protocols differs from before, with the introduction of "loops." These are cycles of activity consisting of the administration of $1 \mathrm{mg}$ adrenaline, 10 ventilation cardiac compression sequences, and attempts at advanced airway care and venous access. Other interventions are dependent on the specific rhythm. The protocols state that adrenaline should be given repeatedly to produce maximal vasoconstriction, diverting blood flow produced by cardiopulmonary resuscitation to the coronary and cerebral circulation.

The protocol for ventricular fibrillation (and pulseless ventricular tachycardia) emphasises the role of early and rapid defibrillation. Previous guidelines have advised that basic life support should begin before and continue between each defibrillation. The new protocols advise that the first three direct current shocks ( $200 \mathrm{~J}, 200 \mathrm{~J}, 360 \mathrm{~J}$ ) should be given in quick succession and without interruption, preferably without removing the defibrillator paddles from the chest.

\section{Lignocaine and bicarbonate: no longer first line treatments}

Although lignocaine has been hallowed by tradition as a useful drug in treating ventricular fibrillation, there is little literature to support this practice and there is experimental evidence that it may make defibrillation more difficult. ${ }^{8}$ No place therefore exists for its routine use, and it joins other drugs, such as sodium bicarbonate, that are for consideration only in prolonged arrest (after three loops).

The protocols for asystole and electromechanical dissociation are very similar to those currently in use in Britain, with minor alterations such as the dose of atropine $(3 \mathrm{mg}$, one dose only) and clearer advice on the indications for pacing. High dose adrenaline $(5 \mathrm{mg})$ should also be considered.

Criticisms of the advanced guidelines are minor. Some emphasis might have been given to hypoxia as a cause of asystole (asthmatic patients arrest in asystole), and practical instructions on how to diagnose the specific causes of electromechanical dissociation would have been helpful.

The ethical issue of when to stop resuscitation is included in the guidelines and, although each case must be judged on its merits, there are clear statements when there is little chance of survival. Similarly, hospitals need to have policies of when to start resuscitation. A study in this week's journal ( $p$ 1577) assesses the awareness of ward nurses of "do not resuscitate" orders written in patients' notes. In about a quarter of patients nurses either did not know the resuscitation status of the patient or got it wrong. The issue of "do not resuscitate" orders is a moral, ethical, and legal minefield, but clearly there are patients who are unlikely to benefit from cardiopulmonary resuscitation. Len Doyal and Daniel Wilsher provide guidance 
through this minefield and suggests guidelines on withholding resuscitation ( $p$ 1593)..$^{10}$

The working parties have had the courage to give a clear message on the need for training. They recommend that basic skills in life support should be taught widely-for example, in schools and as part of driving tests. The advice on training in hospitals and medical schools echoes that of the Royal College of Physicians," but how many junior staff have recently received "compulsory training and retraining"? A study by David and Prior-Willeard shows poor resuscitation skills in the doctors who would probably be in charge of resuscitations on hospital wards ( $p$ 1578). ${ }^{12}$ Minor deviations from perfection are acceptable, but it is difficult to justify poor knowledge of current national guidelines, especially in doctors about to sit the final examination for membership of the Royal College of Physicians.

Another paper in this week's journal reaffirms the need for regular training in cardiopulmonary resuscitation, as without practice such skills soon decay. ${ }^{13}$ Such training is very labour intensive and is often carried out by a few enthusiasts without much support or recognition. There will be no improvement in resuscitation skills until such training attracts adequate resources.

To the best parts of current practice the working parties have added applied science, considered practical requirements, and produced sensible and workable guidelines. If you haven't had training in advanced life support recently, then shouldn't you be getting it soon?

JIM WARDROPE Consultant in accident and emergency medicine FRANCIS MORRIS

Northern General NHS Trust, Consultant in accident and emergency medicine

Herries Road,

Sheffield S5 7AU

1 Basic Life Support Working Party of the European Resuscitation Council. Guidelines for basic life support. Resuscitation 1992;24:103-10.

2 Advanced Life Support Working Party of the European Resuscitation Council. Guidelines fo advanced life support. Resuscitation 1992;24:111-21.

3 European Resuscitation Council Basic Life Support Working Group. Guidelines for basic life support. $B M F$ 1993;306:1587-9.

4 European Resuscitation Council Working Party. Adult advanced cardiac life support: the European Resuscitation Council guidelines 1992 (abridged). BM 1993;306:1589-93.

5 Emergency Cardiac Care Committee and Subcommittees, American Heart Association. Adult basic life support. JAMA 1992;268:2184-98.

6 Caldwell G, Millar G, Quinn E, Vincent R, Chamberlain DA. Simple mechanical methods fo cardioversion: defence of the precordial thump and cough version. $B M F$ 1985;291:627-30.

7 Evans T. $A B C$ of resuscitation. London: British Medical Journal, 1986.

8 Chamberlain DA. Lignocaine and bretylium as adjuncts to electrical defibrillation. Resuscitation 1991;22:167-72.

9 Jones A, Peckett W, Clark E, Sharpe C, Krimholtz S, Russell M, et al. Nurses' knowledge of the resuscitation status of patients and action in the event of cardiorespiratory arrest. $B M$ resuscitation sta

10 Doyal L, Wilsher D. Withholding cardiopulmonary resuscitation: proposals for formal guidelines. $B M F$ 1993;306:1593-6.

11 Royal College of Physicians of London. Resuscitation from cardiopulmonary arrest, training and organisation. London: RCP, 1987.

12 David J, Prior-Willeard PFS. Resuscitation skills of MRCP candidates. BMf 1993;306:1578-9.

13 Berden HJJM, Willems FF, Hendrick JMA, Pijls NHJ, Knape JTA. How frequently should basic cardiopulmonary resuscitation training be repeated to maintain adequate skills? $B M \mathcal{F}$ 1993;306: 1576-7.

\section{The Osteopaths Bill}

\section{What it means for medicine}

Having had its third reading in the Commons on 7 May, $\mathrm{Mr}$ Malcolm Moss's Osteopaths Bill is now in the House of Lords. Barring unforeseen accidents, it will shortly receive the Queen's assent and become law. Lord Walton is steering its passage through the Lords, as he steered an earlier attempt in the previous parliamentary session, with great authority. Since there is undoubtedly cross party support in the Lords and little, if any, opposition it will be surprising now if the bill is not passed.

Osteopathy will then become the first branch of complementary medicine to be formally recognised and regulated in Britain. For the first time it will not be possible for anyone who chooses to describe himself or herself as an osteopath to do so. The model selected for this purpose is essentially the same as that adopted in $\mathbf{1 8 5 8}$ for the medical profession and more recently for dentistry. After a transitional period the only route to entry on the register will be by a course of professional training prescribed by a new General Osteopathic Council of 24 members, comprising 12 elected osteopaths, four representatives of the relevant educational institutions, and eight members appointed by the Privy Council. Seven of these eight will be lay members representing the interests of patients and one will be a registered medical practitioner appointed after consultation with the conference of medical royal colleges. It will thus be a much more compact body than the General Medical Council with, proportionately, a larger lay element.

The council will be responsible not only for prescribing professional training but also for maintaining the register and dealing with problems created by sickness or unacceptable conduct. This will include conduct that is judged to fall below acceptable standards of competence as well as of personal behaviour. In that sense it is, I believe, more appropriate than the emphasis on etiquette in the corresponding medical legislation. It will be illegal for anyone not on this register to describe himself or herself as an osteopath.

What does it all mean for medicine? In a narrow sense the answer may be not much, at least initially. No doctor will be obliged to refer any patient to an osteopath, but some of the barriers for those who wish to do so will be removed. Most people who receive osteopathic treatment now do so of their own volition and pay for it out of their own pockets. They and their doctors will have a new and necessary assurance that the osteopath to whom they go is properly trained and professionally accountable. Direct payment is likely to remain by far the dominant mode, although there will now be increased pressure on private insurers to cover osteopathic treatment. There is no reason in principle why some osteopathy should not be covered within the NHS. The barriers are likely to be financial, except possibly for those general practitioner fundholders and district purchasers who judge that for some conditions osteopathy is more cost effective than orthodox medical treatment.

Osteopathy would not have got so far as a private member's bill without (as an absolute minimum) a lack of opposition from doctors, nurses, and physiotherapists. In fact, it received active support. But what about the long list of other complementary treatments, from acupuncture and aromatherapy to reflexology? Behind the Osteopaths Bill lay the report of a King's Fund working party chaired by Sir Thomas Bingham, now Master of the Rolls. A similarly constituted working party has now produced a report on chiropractic, directly modelled on Mr Moss's Osteopaths Bill. The two cases are closely comparable, and there are good arguments for getting 